\title{
The skin as the source of Acinetobacter and Moraxella species occurring in blood cultures
}

\author{
MAYSOON S. AL-KHOJA AND J. H. DARRELL \\ From the Department of Bacteriology, Royal Postgraduate Medical School, Hammersmith Hospital, \\ Du Cane Road, London W12 OHS, UK
}

SUMMARY A study was made of the flora of the skin in hospital inpatients and healthy people to demonstrate the presence of non-fermenting Gram-negative rods of the Acinetobacter and Moraxella group. These organisms were found to be present on the skin of $34.3 \%$ of inpatients and occurred even more commonly in those patients with kidney disease. It was also present on the skin of $20 \%$ of a group of healthy members of staff. This rather high rate of skin carriage is thought to account for the not infrequent occurrence of this organism in blood cultures.

In 1975 our laboratory processed approximately 2700 blood cultures. In 31 of the 372 yielding growth, the organism was a non-fermenting Gramnegative rod of the Morexella-Acinetobacter group. Only two of these isolates appeared to be associated with clinical evidence of infection, the organisms occurring in all three bottles of these blood cultures whereas the others, probable contaminants, occurred usually in one only and never in all the bottles. After exclusion of any possible contamination of the media used during preparation or processing in the laboratory, 70 patients were studied to establish the source of this probable contamination. Thirty healthy members of the laboratory staff were studied as a normal non-hospitalised control group.

\section{Material and methods}

Contact plates (Sterilin Limited) of MacConkey media (Oxoid CM7b) were used to sample the skin of the antecubital fossa before and after disinfection with isopropyl alcohol (Medi Swabs). This site was chosen because it is the usual site from which blood is taken for culture. Eosin methylene blue agar was tested as a selective medium for Acinetobacter anitratus in the contact plates as suggested by Taplin and Rebell (1963) but most strains gave scanty growth and it was abandoned in favour of MacConkey media. After overnight incubation at $37^{\circ} \mathrm{C}$ suspected colonies, namely non-lactose fermenting small coliform colonies, oxidase negative in the case of Acinetobacter and oxidase positive in the case of Moraxella, were further identified according to the

Received for publication 8 November 1978 methods of Cowan and Steel (1977). In addition, ammonium salt sugars, Tween 80 Sierra medium and Nagler plates to demonstrate lecithinase activity were inoculated. Sensitivity tests were performed on lysed blood agar plates using Stokes' method with the Oxford staphylococcus (NCTC 6571) as the control organism.

\section{Results}

Isolates of Acinetobacter were speciated according to their biochemical reactions (Table 1), many of which were used by Snell (1973). It is difficult to

Table 1 Biochemical reactions of Acinetobacter and Moraxella species

\begin{tabular}{lrll}
\hline Test & \multicolumn{2}{c}{$\begin{array}{c}\text { No. of strains } \\
\text { in each test }\end{array}$} & \\
\cline { 2 - 4 } & A. lwoffing a positive reaction \\
\cline { 2 - 4 } & 16 & A. anitratus & Moraxella \\
\hline No. of strains tested & 0 & 6 & 8 \\
Oxidase & 11 & 0 & 8 \\
O/F test-negative & 5 & 0 & 2 \\
$\quad$ alkali produced & 0 & 0 & 6 \\
oxidative & 0 & 6 & 0 \\
Motility at 22 ${ }^{\circ}$ C & 0 & 0 & 0 \\
Peptone water glucose & 0 & $6 \dagger$ & 0 \\
Peptone water lactose 10\% & 12 & 6 & 0 \\
Amm. salt sugar (xylose) & 11 & 6 & 0 \\
Amm. salt sugar (glucose) & 6 & 3 & 0 \\
Simmons citrate & 3 & 0 & 0 \\
Lecithinase production & 16 & 6 & 0 \\
Tween 80 & 7 & 3 & 2 \\
Urea & 0 & 6 & 0 \\
Malonate* & & & 0 \\
\hline
\end{tabular}

$\mathbf{O} / \mathbf{F}=$ Oxidation-fermentation test.

+ Reaction positive after 2-4 days' incubation.

* Not tested by us. 
identify Moraxella to species level. The reactions of the genus are given for comparison with Acinetobacter species. The $\mathrm{O} / \mathrm{F}$ test and the reaction in peptone water glucose are the tests which most readily distinguish the two Acinetobacter species in routine practice (King and Phillips, 1978). We were not able to confirm the characteristic smell of $A$. lwoffii recorded by these authors in some strains.

The result of this investigation is summarised in Table 2. It was found that patients with kidney disease showed higher carriage rates than other patients (Table 2). Occasional hospitalised patients

Table 2 Skin carriage of non-fermenting rods in hospital patients and healthy staff

\begin{tabular}{lllc}
\hline Subjects & Total & \multicolumn{2}{l}{$\begin{array}{l}\text { ACI-MOR group } \\
\text { isolated from skin }\end{array}$} \\
\cline { 3 - 4 } & & No. & $\%$ \\
\hline Hospitalised patients & & & \\
With kidney disease & 23 & 11 & $47 \cdot 8$ \\
With other conditions & 47 & 13 & $27 \cdot 6$ \\
Total & 70 & 24 & $34 \cdot 3$ \\
Healthy staff & 30 & 6 & $20 \cdot 0$ \\
\hline
\end{tabular}

showed persistent skin carriage over periods of up to nine weeks and longer. Some acquired the organisms during their stay in hospital. A group of five patients in our intensive care unit were also investigated by sampling one hour after cardiac operation for which extensive skin preparation had been carried out. One patient yielded a few colonies of Acinetobacter from the antecubital fossa immediately postoperatively. Skin carriage of Acinetobacter spp was commoner than carriage of Moraxella spp, and the numbers of isolates of $A$. lwoffii were greater than those of $A$. anitratus (Table 3).

Table 3 Frequency of skin carriage of Acinetobacter and Moraxella in hospital patients and healthy staff

\begin{tabular}{lllll}
\hline Subjects & $\begin{array}{l}\text { Total } \\
\text { number }\end{array}$ & A. lwoffii & A. anitratus & $\begin{array}{l}\text { Moraxella } \\
\text { species }\end{array}$ \\
\hline Patients & 70 & 12 & 4 & 8 \\
Controls & 30 & 4 & 2 & 0 \\
\hline
\end{tabular}

The figures given for skin carriage in the tables are before skin preparation. After skin preparation the numbers of these organisms were greatly reduced but small numbers (one to three) of colony-forming units persisted in spite of skin preparation in three of 16 patients carrying Acinetobacter.

The results of sensitivity tests are summarised in Table 4.
Table 4 Antibiotic sensitivity of Acinetobacter and Moraxella species

\begin{tabular}{llll}
\hline Antibiotic & \multicolumn{2}{l}{ Number of strains sensitive } \\
\cline { 2 - 4 } & A. lwoffii & A. anitratus & $\begin{array}{l}\text { Moraxella } \\
\text { species }\end{array}$ \\
\hline No. of strains tested & 16 & 6 & 8 \\
Penicillin 1 unit/disc & 0 & 0 & 8 \\
Ampicillin $10 \mu \mathrm{g} / \mathrm{disc}$ & 7 & 4 & 8 \\
Trimethoprim $2 \cdot 5 \mu \mathrm{g} / \mathrm{disc}$ & 4 & 0 & 3 \\
Sulphafurazole $200 \mu \mathrm{g} / \mathrm{disc}$ & 11 & 4 & 8 \\
Cephaloridine $5 \mu \mathrm{g} / \mathrm{disc}$ & 1 & 0 & 8 \\
Gentamicin $10 \mu \mathrm{g} / \mathrm{disc}$ & 16 & 6 & 8 \\
\hline
\end{tabular}

\section{Discussion}

The skin was first studied as a potential reservoir of the Acinetobacter group by Taplin and Rebell in 1963. At this time they were known as Mima polymorpha (Acinetobacter lwoffii) and Herrellea (Acinetobacter anitratus). They were found to occur on skin surfaces at rates of $10 \%$ and $25 \%$ respectively. Dadswell (1976) studied Acinetobacter and similar organisms in ear infections. He found that while the majority of his isolates played little or no part in the infective process, presumably being commensals in the skin auditory meatus, a few were associated with acute or chronic ear disease. There have been several cases of reported bacteraemia (CDR Reports, 1978) caused by Acinetobacter. Van de Torregrosa and Ortiz (1961) reported cases of meningitis in children due to Acinetobacter and Moraxella. Our study was directed specifically at deciding the probable significance of isolates from blood cultures. It appears that when these organisms are isolated from blood cultures of patients with no clinical evidence of bacteraemia, it should be remembered that they are common skin commensals and the growth could represent contamination, especially as our studies show that Acinetobacter withstood the routine skin disinfection in $18.7 \%$ of patients carrying the organism. In none of the patients studied in this series was the organism felt to be playing a pathogenic role in spite of the fact that one had a positive blood culture in addition to the positive skin culture. However, occasional bacteraemic episodes occur in our practice usually in compromised patients.

\section{References}

Cowan, S. T. (1977). Cowan and Steel's Manual for the Identification of Medical Bacteria, 2nd edition. Cambridge University Press, London.

Dadswell, J. V. (1976). Acinetobacter and similar organisms in ear infections. Journal of Medical Microbiology, 9, 345-353. 
King, A., and Phillips, I. (1978). The identification of pseudomonads and related bacteria in a clinical laboratory. Journal of Medical Microbiology, 11, 165-177.

Snell, J. J. S. (1973). The Distribution and Identification of Non-fermenting Bacteria (PHLS Monograph Series, No. 4). HMSO, London.

Taplin, D., and Rebell, G. (1963). The human skin as a source of Mima-Herellea infections. Journal of the American Medical Association, 186, 952-954.
Van de Torregrosa, M., and Ortiz, A. (1961). Severe infections in children due to rare gram-negative bacilli (Mima polymorpha and Bacillus anitratum). Journal of Pediatrics, 59, 35-41.

Requests for reprints to: Dr J. H. Darrell, Department of Bacteriology, Royal Postgraduate Medical School, Hammersmith Hospital, Du Cane Road, London W12 OHS, UK. 